\title{
Proteomic identification of down-regulation of oncoprotein DJ-1 and proteasome activator subunit 1 in hepatitis $B$ virus-infected well-differentiated hepatocellular carcinoma
}

\author{
DAOHAI ZHANG ${ }^{1,2 *}$, SENG GEE LIM ${ }^{3}$ and EVELYN S.C. KOAY $Y^{1,2 *}$ \\ ${ }^{1}$ Department of Laboratory Medicine, National University Hospital; Departments of ${ }^{2}$ Pathology, and \\ ${ }^{3}$ Medicine, Yong Loo Lin School of Medicine, National University of Singapore, Singapore 119074
}

Received February 12, 2007; Accepted April 2, 2007

\begin{abstract}
Hepatocellular carcinoma (HCC) is a common malignant tumour. Development of HCC is a multi-step process from well-differentiated (G1), moderately differentiated (G2) to poorly differentiated (G3) phenotype. The early molecular modulators causing the onset of hepatocarcinogenesis are not fully understood. In the present study, we conducted comparative proteomics to analyze the differential proteome of G1 tumour and adjacent non-tumour tissues, with aims to identify the molecules as early tumour markers and to understand the early molecular events involved in initiation of tumorigenesis in hepatitis B virus (HBV)-infected G1 tumour. Differentially expressed proteins were identified by MALDITOF/TOF tandem mass spectrometry and NCBInr database interrogation. A total of 15 differentially expressed proteins with diverse biological functions were identified. Among these, 4 proteins were down-regulated, whereas the other 11 proteins were up-regulated in the G1 tumours. Two proteins, Proteasome activator subunit 1 (PA28 $\alpha$ ) and DJ-1, were firstly found to be down-regulated in HBV-infected G1 tumours. Down-regulations of these two proteins were further validated by Western blotting and immunohistochemistry in a panel of clinical specimens. These findings elucidate, at least in part, the molecular events underlying the mechanism and
\end{abstract}

Correspondence to: Dr Daohai Zhang, Molecular Diagnosis Centre, Department of Laboratory Medicine, National University Hospital, 5 Lower Kent Ridge Road, Singapore 119074

E-mail: daohai_zhang@nuh.com.sg

${ }^{*}$ Co-corresponding authors

Abbreviations: HCC, hepatocellular carcinoma; PA28 $\alpha$, proteasome activator subunit 1; DJ-1, Parkinson disease (autosomal recessive, early onset) 7; AFP, $\alpha$-fetoprotein; HBV, hepatitis B virus; $\mathrm{HCV}$, hepatitis $\mathrm{C}$ virus; 2-DE, 2-dimensional electrophoresis

Key words: hepatocellular carcinoma, proteomics, well differentiated, PA28 $\alpha$, DJ-1, hepatitis B virus the potential roles of DJ-1 and PA28 $\alpha$ in the onset of hepatocarcinogenesis.

\section{Introduction}

Hepatocellular carcinoma (HCC) develops from a chronic inflammatory liver disease caused by hepatitis $\mathrm{B}$ or $\mathrm{C}$ virus, alcohol abuse, exposure to carcinogen such as aflatoxin B1, and genetic alteration, etc. (1). The high incidence of death of patients with HCC is mainly due to the lack of a valuable prognostic indicator and effective treatment. There are a few biomarkers for clinical diagnosis in HCC: $\alpha$-fetoprotein (AFP), des- $\gamma$-carboxy prothrombin plasma abnormal prothrombin, the L3 fraction (AFP-L3), isozyme of alkaline phosphatase (variant ALP), and isozyme of $\gamma$-glutamyl transpeptidase (novel $\gamma$-GTP) (2). However, considering the sensitivity, specificity and operability, only AFP is a useful marker in the diagnosis of HCC, especially HBV-associated HCC. Nevertheless, the AFP level is normal in up to $30 \%$ of patients with liver cancer, and other factors including early childhood liver damage from hepatitis, or tumours of the testis can also raise the AFP level. Therefore, as an HCC tumour marker, the AFP test has minimal clinical significance and must be interpreted together with a proper imaging diagnosis of the liver.

Recently, the proteomics approach has been widely used in discovering tumour-associated biomarker/drug targets and in deciphering the molecular mechanism underlying the tumorigenesis. In HCC, many potential markers have been identified from clinical tissue specimens using different proteomic approaches, including laser capture microdissection (LCM), isotope-coded affinity Tag (ICAT), two-dimensional gel electrophoresis (2-DE), two-dimensional difference gel electrophoresis (2-D-DIGE) and mass spectrometry (3-9). Such analyses have revealed the complicated molecular repertoire of markers which are involved in a variety of biological functions including signal transduction, metabolism and apoptosis. Unfortunately, only a few of the protein markers have been validated in clinical specimens due to the lack of available antibodies or due to the large-scale labour-intensive work involved in the validation process. Among them, alteration of aldehyde dehydrogenase isozyme closely correlated to HCC (10), and human aldose reductaselike protein-1 was suggested to be a strong candidate as an 
immunohistochemical diagnostic marker (6). Further, the Hsp27 expression correlated with the level of AFP but not with tumour stage (5), although it was considered to be a potential serum biomarker in patients with HCC (11). Other potential markers include $\alpha$-enolase whose level correlated to $\mathrm{HCV}$-infected HCC tumour progression (12) and protein $\mathrm{C} 3 \mathrm{a}$ whose expression was elevated in patients with HCV-related HCC (13). Nevertheless, clinical validation and application of the tumour-marker candidates are an urgent requirement in cancer translational interface.

HCC is developed from a multi-step process based on histologic changes: tumour cells are initially well differentiated (grade 1, G1), and then progress to moderately differentiated (grade II, G2) and poorly differentiated (grade III, G3) types with high proliferation rates. Evidence has shown that the proteome profiles of HCC tumours are grade-dependent. In HCC, cytokeratin 19 (CK19) was highly expressed in the late stage of tumours (G3) and strongly related to metastatic behaviour (14). $\alpha$-enolase was strongly expressed in G3 tumours compared with G1 and G2 tumours (12), and glutamine synthetase was highly expressed in HCV-infected G1 tumours (15). Recently, protein Hcc-2 was found to be upregulated in G3 tumours but unchanged in G1 tumours (16). In addition, proteome profiles of HCC are also virus-dependent. Previous study demonstrated that HCC proteome and gene expression profiling vary between $\mathrm{HBV}$ - and $\mathrm{HCV}$-infected HCC tumours $(8,17)$. Kim et al found that Hsp27 was downregulated in $\mathrm{HBV}$-related $\mathrm{HCC}$, but up-regulated in $\mathrm{HCV}$ related $\mathrm{HCC}$, whereas ECH1 was highly expressed in $\mathrm{HCV}$ infected HCC, but decreased in HBV-infected HCC tumours with no clarification of tumour grade (8). As such, the HCC specimens for proteomics study should be carefully selected when addressing the relevance of molecular alterations with specific aetiology and/or histopathological features.

In the present study, we analyzed the differential proteome profiles between HBV-infected G1 tumour and adjacent nontumour tissues in order to identify the potential molecules as early tumour markers and to understand the early molecular events involved in initiation of tumorigenesis. We have identified 15 dysregulated proteins with varieties of biological functions by MALDI-TOF/TOF tandem mass spectrometry and NCBInr database interrogation. Two proteins including oncoprotein DJ-1 and proteasome activator subunit 1 (PA28 $\alpha$ ) were, for the first time, found to be down-regulated in the HBV-infected G1 tumour. Their roles in hepatocarcinogenesis and potentials as early biomarkers for cancer diagnosis are discussed.

\section{Materials and methods}

Tissue specimens. Six pairs of human HBV-infected (G1) HCC frozen tumour tissues and the adjacent non-tumour tissues were provided by Professor SG Lim, Department of Medicine, National University Hospital. The HCC tissue collections were obtained under informed consent and were approved by the ethics committee of the hospital. These tumour specimens were diagnosed as well differentiated (G1) by pathological examination, according to the method described by Desmet et al (18). These tumour/non-tumour tissues were used for proteome analysis.
Protein extraction and protein separation by 2-dimensional electrophoresis $(2-D E)$. For each sample, the frozen liver tissue was transferred to a pre-cooled mortar, ground to powder in liquid nitrogen, and solubilized in Tissue Protein Extraction Reagent ${ }^{\circledR}$ (Pierce, Rockford, IL, USA) according to the manufacturer's protocol. Samples were cleaned up using a PlusOne 2-D Clean-up kit (GE Healthcare, San Francisco, CA, USA) and re-dissolved in sample buffer (7 M urea, $2 \mathrm{M}$ thiourea, $4 \%$ CHAPS, $1 \%$ Mega-10, 0.5\% Triton $\mathrm{x}-100,50 \mathrm{mM}$ dithiothreitol, 1\% IPG buffer ( $\mathrm{pH} \mathrm{4-7)}$ and $2 \mathrm{mM}$ tributyl phosphine). Protein concentrations were determined using the PlusOne 2-D quantitation kit (GE Healthcare).

2-DE and image analysis were performed as described previously (19). Briefly, equal amounts (120 $\mu \mathrm{g})$ of proteins from tumour and matched non-tumour tissues were separately mixed with $350 \mu 1$ of rehydration solution containing $7 \mathrm{M}$ urea, $2 \mathrm{M}$ thiourea, $4 \%$ CHAPS, $0.5 \%$ IPG buffer ( $\mathrm{pH} 4-7$ ), and rehydrated for $12 \mathrm{~h}$ at $20 \mathrm{~V}$ using the Immobiline IPG DryStrips (180 mm, pH 4-7) (GE Healthcare). Proteins were isoelectrically focused at $500 \mathrm{~V}$ for $1 \mathrm{~h}, 2000 \mathrm{~V}$ for $1 \mathrm{~h}, 4000 \mathrm{~V}$ for $2 \mathrm{~h}$ and $8000 \mathrm{~V}$ for $6 \mathrm{~h}$ on an Ettan IPGphor electrophoresis system (GE Healthcare). After equilibration for 2x15 min in equilibration buffer, IPG strips were applied for SDS-PAGE (12\%) using a PROTEAN ${ }^{\circledR}$ II xi Cell system (Bio-Rad, Hercules, CA, USA). The separated proteins were visualized by silver staining (19).

Image acquisition and analysis. Silver-stained gels were scanned using the Personal SI densitometer (Molecular Dynamics, Sunnyvale, CA, USA). Spot detection, matching and expression levels of proteins on 2-DE gels were quantitatively analyzed, based on the spot intensities, using the ImageMaster 2D Elite software (GE Healthcare). Differences in expression levels between paired samples were analyzed by Student's t-test. Protein spots showing at least 2-fold changes $(\mathrm{p}<0.05)$ in intensity were picked for trypsin digestion and MALDI-TOF/TOF MS/MS analysis.

Trypsin in-gel digestion and mass spectrometry. The protein spots were manually excised from the gel and digested with trypsin, as described previously (19). After being dehydrated with acetonitrile and dried in a SpeedVac centrifuge, the gels were re-swelled with 20-40 $\mu \mathrm{l}$ digestion solution containing $20 \mathrm{mM}$ ammonium bicarbonate and $20 \mathrm{ng} / \mu \mathrm{l}$ of sequencing grade trypsin (Promega, Madison, WI, USA) and treated at $37^{\circ} \mathrm{C}$ overnight. The resultant peptides were extracted, desalted with ZipTip $\mathrm{C}_{18}$ columns (Millipore Corporation, Bedford, MA, USA) and eluted with $2.5 \mu \mathrm{l}$ of $50 \%$ ACN containing $0.5 \%$ TFA. Peptide mass spectra were obtained by the ABI 4700 proteomics analyzer MALDI-TOF/TOF MS/MS (Applied Biosystems, Framingham, MA, USA), as described (20). Known contamination peaks such as keratin and autoproteolysis peaks were removed prior to the database search. Protein identification was performed using the MASCOT software (Matrix Science, London, UK) and all tandem mass spectra were searched against the NCBInr database, with a mass accuracy of within $200 \mathrm{ppm}$ for the mass measurement, and within $0.5 \mathrm{Da}$ for MS/MS tolerance window. Searches were performed without constraining protein molecular weight $(\mathrm{Mr})$ or isoelectric point $(\mathrm{pI})$ and species, and allowing for 
Table I. Summary of identified proteins with MALDI-TOF/TOF tandem mass spectrometry. ${ }^{2}$

\begin{tabular}{|c|c|c|c|c|c|c|c|}
\hline Spot no. & Protein ID & Swiss-Prot & $\mathrm{Mr} / \mathrm{pI}$ & Score & $\begin{array}{l}\text { MS/MS peptide/ } \\
\text { matched peptide }\end{array}$ & $\begin{array}{c}\text { Relative level } \\
\text { (tumour/non-tumour) }\end{array}$ & $\begin{array}{c}\text { Sequence } \\
\text { coverage (\%) }\end{array}$ \\
\hline 1 & $\begin{array}{l}\text { Heat shock protein } 27 \\
\text { (HSP27) }\end{array}$ & P04792 & $22.4 / 7.83$ & 358 & $5 / 13$ & 2.23 & 52 \\
\hline 2 & $\begin{array}{l}\text { Chloride intracellular } \\
\text { channel } 1 \text { (CLIC1) }\end{array}$ & O00299 & $26.5 / 4.95$ & 171 & $3 / 9$ & 2.16 & 35 \\
\hline 3 & $\begin{array}{l}\text { Glutathione S-transferase } \\
\text { (GST) }\end{array}$ & P09211 & $23.6 / 5.43$ & 344 & $3 / 14$ & 3.22 & 57 \\
\hline 4 & Regucalcin (RGN) & Q15493 & $33.8 / 5.89$ & 105 & $2 / 10$ & 0.23 & 33 \\
\hline 5 & $\begin{array}{l}\text { Inorganic pyrophosphatase } \\
\text { (PPA) }\end{array}$ & Q15181 & $33.1 / 5.54$ & 87 & $2 / 13$ & 2.78 & 32 \\
\hline 6 & Ketohexokinase (KHK) & P50053 & $25.5 / 5.99$ & 141 & $2 / 9$ & 2.35 & 40 \\
\hline 7 & $\begin{array}{l}\text { 3-Hydroxyanthranilate } \\
\text { 3,4-dioxygenase (HAAO) }\end{array}$ & P46952 & $32.7 / 5.72$ & 83 & $2 / 9$ & 2.51 & 32 \\
\hline 8 & $\begin{array}{l}\text { Enoyl-CoA hydratase } \\
\text { (ECH1) }\end{array}$ & Q13011 & $31.8 / 8.34$ & 169 & $2 / 10$ & 2.19 & 38 \\
\hline 9 & $\begin{array}{l}\text { Nucleoside diphosphate } \\
\text { kinase (NM23-H1) }\end{array}$ & P15531 & $19.8 / 5.42$ & 396 & $6 / 16$ & 2.08 & 51 \\
\hline 10 & Tubulin $\alpha-6$ (TUBA6) & Q9BQE3 & $50.7 / 4.96$ & 307 & $5 / 14$ & 2.78 & 33 \\
\hline 11 & Tubulin B-chain (TUBB) & P07437 & $50.3 / 4.78$ & 118 & $3 / 13$ & 2.15 & 27 \\
\hline 12 & $\begin{array}{l}\text { Apolipoprotein A-1 } \\
\text { (APOA1) }\end{array}$ & P02647 & $30.7 / 5.56$ & 130 & $5 / 17$ & 2.59 & 40 \\
\hline 13 & $\begin{array}{l}\text { Proteasome activator } \\
\text { subunit } 1 \text { isoform } 2(\mathrm{PA} 28 \alpha)\end{array}$ & Q06323 & $28.7 / 6.28$ & 104 & $3 / 9$ & 0.45 & 17 \\
\hline 14 & DJ-1 & Q99497 & $20 / 6.33$ & 109 & $2 / 9$ & 0.32 & 22 \\
\hline 15 & $\begin{array}{l}\text { Triosephosphate } \\
\text { isomerase (TPI1) }\end{array}$ & P60174 & 26.7/7.1 & 81 & $2 / 6$ & 0.38 & 23 \\
\hline
\end{tabular}

${ }^{a}$ The Mowse score of $>75$ was considered as significant $(\mathrm{p}<0.05)$.

carbamidomethylation of cysteine and partial oxidation of methionine residues. Up to one missed tryptic cleavage was considered for all tryptic-mass searches. Protein scores $>75$ were considered to be significant $(\mathrm{p}<0.05)$.

Immunoblot analysis. Twenty $\mu \mathrm{g}$ of total proteins from six pairs of HCC samples were separated by $12 \%$ SDS-PAGE and electro-transferred onto poly-vinylidene difluoride (PVDF) membranes by the SemiDry apparatus (Bio-Rad). The membranes, after blocking with 5\% nonfat dry milk in TBS-T buffer (Tris-buffered saline, $0.1 \%$ Tween-20), were probed with one of the following antibodies: mouse monoclonal anti-human DJ-1 (Zymed ${ }^{\circledR}$ Laboratories, South San Francisco, CA, USA), rabbit polyclonal anti-human PA28 $\alpha$ (Calbiochem $\left.{ }^{\circledR}\right)$, and incubated at $4^{\circ} \mathrm{C}$ overnight. $\beta$-actin signal was detected by a mouse monoclonal anti-human $B$-actin antibody (SigmaAldrich, St. Louis, MO, USA) and used as an internal control. Goat anti-rabbit IgG horseradish peroxidase $\left(Z_{y M E D}{ }^{\circledR}\right.$ Laboratories Inc, San Francisco, CA, USA) or goat anti-mouse IgG horseradish peroxidase (Upstate, Lake Placid, NY, USA) were used as secondary antibodies. Protein signals were detected using SuperSignal ${ }^{\circledR}$ West Pico chemiluminescent substrate (Pierce), and captured with the Multi Genius bio imaging system (Syngene, Frederick, MD, USA). Signal intensity was quantitatively analyzed using the GeneTools software (Syngene).

Immunohistochemistry. Immunohistochemical staining (IHC) of DJ-1 and PA28 $\alpha$ in 15 pairs of paraffin-embedded, HBVinfected G1 tumour and non-tumour tissues were performed 
using the Dako system as previously described $(19,20)$. Briefly, sections of $4-\mu \mathrm{m}$ thickness from both tumour and matched non-tumour tissue blocks were dewaxed, rehydrated and labelled for $60 \mathrm{~min}$ at room temperature with each of the two antibodies after conducting the antigen retrieval: anti-DJ-1 (1:20) and anti-PA28 (1:50), followed by detection with labelled dextran polymer conjugated with peroxidase and $\mathrm{DAB}^{+}$-substrate chromagen solution. Slides were lightly counter-stained with hematoxylin before imaging. The staining levels were scored as negative, weak, moderate and strong based on the staining intensity by the pathologist. Negative and weak stainings were considered as negative whereas moderate and strong stainings were considered as positive.

Statistical analysis. Statistical differences in the levels of PA28 $\alpha$ and DJ-1 in paired tumour and non-tumour tissues were analyzed by Fisher's exact test. $\mathrm{p}<0.05$ at two sides was considered as significant.

\section{Results}

Proteomic analysis of tumour and non-tumour tissues. To identify the potential tumour markers for early diagnostic purpose and elucidate the molecular changes at the early onset of heptocarcinogenesis, we compared the proteome profiles of three pairs of $\mathrm{HBV}$-infected G1 tumour and adjacent non-tumour tissues. With the ImageMaster analysis software, more than 700 spots were detected in each gel, and in total, 35 spots were found to be consistently up- or down-regulated $(>2.0$-fold increase or decrease, Student t-test, $\mathrm{p}<0.05)$ in at least two pairs of specimen. Fig. 1 shows the representative silver-stained 2-DE gel. After MALDI-TOF/TOF tandem mass spectrometry analysis and NCBInr database search

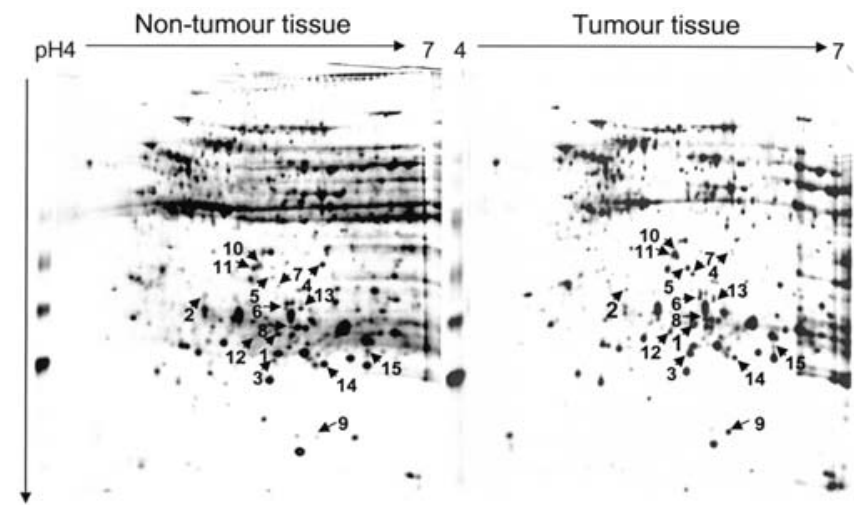

Figure 1. Differential proteome analysis of HBV-infected, well-differentiated HCC tumour and adjacent non-tumour tissues. Total protein lysates were separated by IEF using IPG DryStrip (180 mm, pH 4-7) and SDS-PAGE $(12 \%)$. Silver-stained gels were scanned using the Personal SI densitometer (Molecular Dynamics) and analyzed using the ImageMaster 2D Elite software (GE Healthcare). The locations of identified proteins by MALDI-TOF/TOF tandem mass spectrometry are shown.

using Mascot software, 15 proteins were identified as listed in Table I. Their positions on the 2-DE map are annotated in Fig. 1. Of these identified proteins, 4 (RGN, DJ-1, PA28 $\alpha$ and TPI) were down-regulated, whereas the other 11 were up-regulated, in the HBV-infected G1 tumours. Most of these identified proteins have been reported elsewhere $(3,8,9,21)$, however, two proteins including PA28 $\alpha$ (spot 13) and oncoprotein DJ-1 (spot 14), that were not previously identified in the proteome analysis of $\mathrm{HCC}$, were found to be downregulated in the $\mathrm{HBV}$-infected G1 tumours. Their representative 2-DE spot images with the MS/MS spectrum are shown in Fig. 2 .

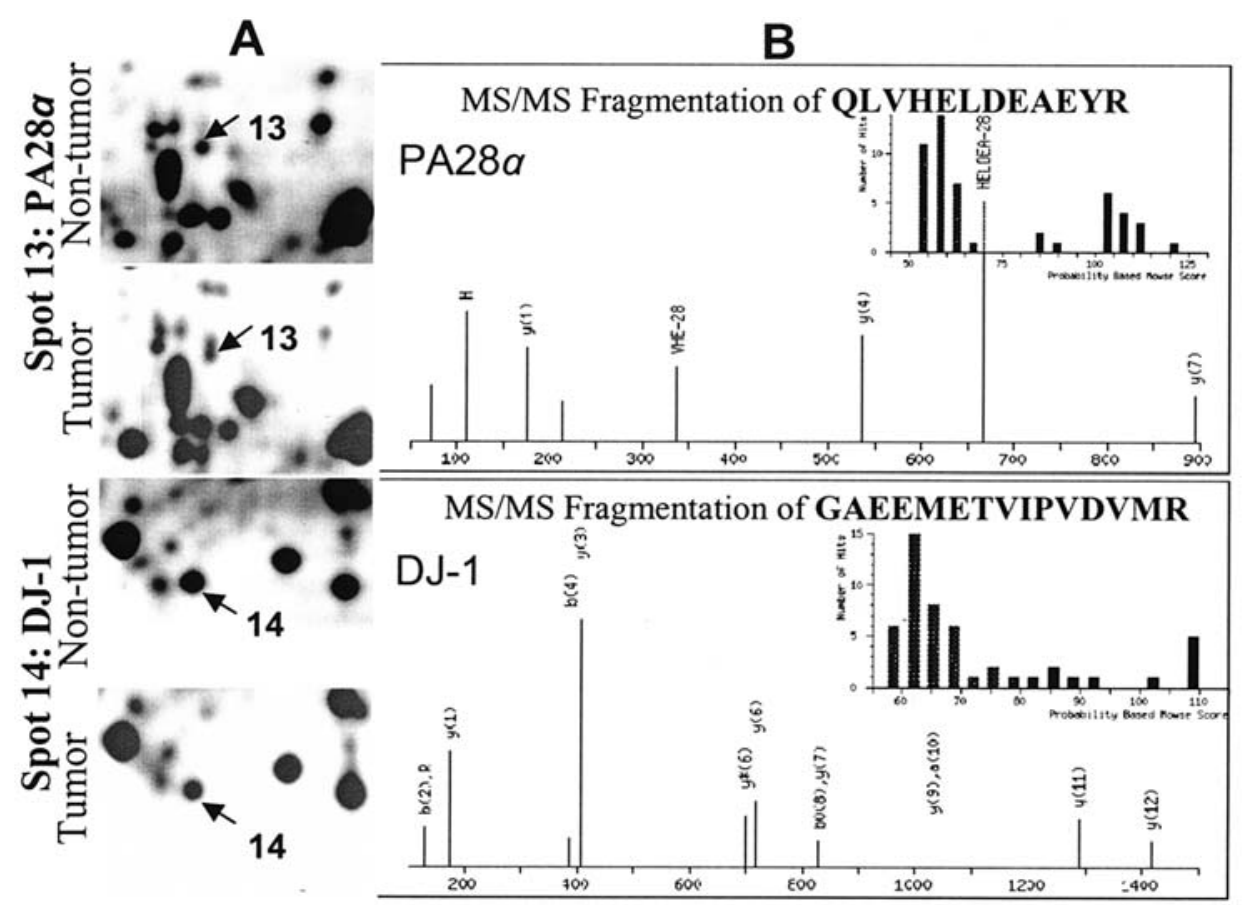

Figure 2. Identification of PA28 $\alpha$ (spot 13) and DJ-1 (spot 14) by tandem mass spectrometry. (A) Zoom images from 2-DE gels showing spots containing PA28 $\alpha$ and DJ-1. (B) MS/MS spectrum of the doubly charged precursor ion with $\mathrm{m} / \mathrm{z} 1501.75$ for peptide ${ }^{199} \mathrm{QLVHELDEAEYR}^{210}$ of PA28 $\alpha$ and the doubly charged precursor ion with $\mathrm{m} / \mathrm{z} 1675.83$ for peptide ${ }^{13}$ GAEEMETVIPVDVMR ${ }^{27}$ of DJ-1. Inserted figure is the Mowse score from database search. 
Table II. Geneontology analysis of identified proteins.

\begin{tabular}{lllr}
\hline Biological function & & \multicolumn{1}{c}{ Protein } & Subcellular localization \\
\hline Anti-apoptosis & Heat shock protein 27 & Cytoplasm & $7 \mathrm{q} 11.23$ \\
& Glutathione S-transferase & Cytoplasm & $11 \mathrm{q} 13.2$ \\
Ca $^{2+}$ transport/signal transduction & Chloride intracellular channel 1 & Nucleus & $6 \mathrm{p} 21.33$ \\
& Regucalcin & Cytoplasm & Xp11.3 \\
Metabolism & Inorganic pyrophosphatase & Cytoplasm & $10 \mathrm{q} 22.1$ \\
& Ketohexokinase & Cytoplasm & $2 \mathrm{q} 23.3$ \\
& 3-Hydroxyanthranilate 3,4-dioxygenase & Cytoplasm & $2 \mathrm{q} 21$ \\
& Enoyl-CoA hydratase & Mitochondria and peroxisome & $19 \mathrm{q} 13.1$ \\
& Triosephosphate isomerase & Cytoplasm & $12 \mathrm{p} 13.31$ \\
Metastasis & Nucleoside diphosphate kinase (NM23-H1) & Nuclear and cytoplasm & $17 \mathrm{q} 21.33$ \\
Structural molecules & Tubulin $\alpha-6$ & Cytoplasm & $12 \mathrm{q} 13.12$ \\
& Tubulin $\beta$-chain & Cytoplasm & $6 \mathrm{p} 21.33$ \\
Transport & Apolipoprotein A-1 & Secreted protein & $11 \mathrm{q} 23.3$ \\
Immune response & Proteasome activator subunit 1 isoform 2 & Proteasome complex & $14 \mathrm{q} 11.2$ \\
Protein folding/Ras protein & DJ-1 & Nuclear and cytoplasm &
\end{tabular}

Functional classification of the tumour marker molecules. To understand the biological relatives of the identified molecules with the onset of tumorigenesis in HBV-infected HCC, the identified 15 proteins were classified by their biological functions with the tools on www.geneontology.org (Table II). In brief, these proteins were functionally involved in enzyme activity (glyco-metabolism, lipid metabolism), immune activity, anti-apoptosis, $\mathrm{Ca}^{2+}$ binding/signal transduction, cell structure, protein folding and metastasis. Of the 15 proteins, eight were cytoplasmic, two were nucleic, one was mitochondrial, one was peroxisomal and one was secreted protein. Furthermore, by analyzing the chromosomal localization of these proteinencoding genes and genomic imbalance, we found a link. For example, the Hsp27 gene, located at 7q11.23, mapped within cancer-related chromosome region 7q11.23 (colon cancer and prostate cancer). On chromosome 1, chromosomal localization of oncogene DJ-1, 1p36.23, is associated with three cancer-related chromosome regions: 1p36 (ductal breast cancer), $1 \mathrm{p} 36$ (prostate cancer-brain cancer susceptibility) and 1p36.11 (liver cancer). Abnormality of these proteins may be involved in a network or oncogenic signal pathways required for the onset of the multi-step process in HCC development.

Immunoblotting and immunohistochemistry. As the reduced expression of the two proteins, PA28 $\alpha$ and DJ-1, has not been identified previously, we further confirmed their differential expression by Western blotting in 6 pairs of HBV-infected G1 tumour and the adjacent non-tumour tissues. As shown in Fig. 3, expression levels of both PA28 $\alpha$ and DJ-1 are shown in a case-dependent manner. However, the overall expression levels in the tumour tissues were lower than those in the matched non-tumour tissues. The levels of both DJ-1
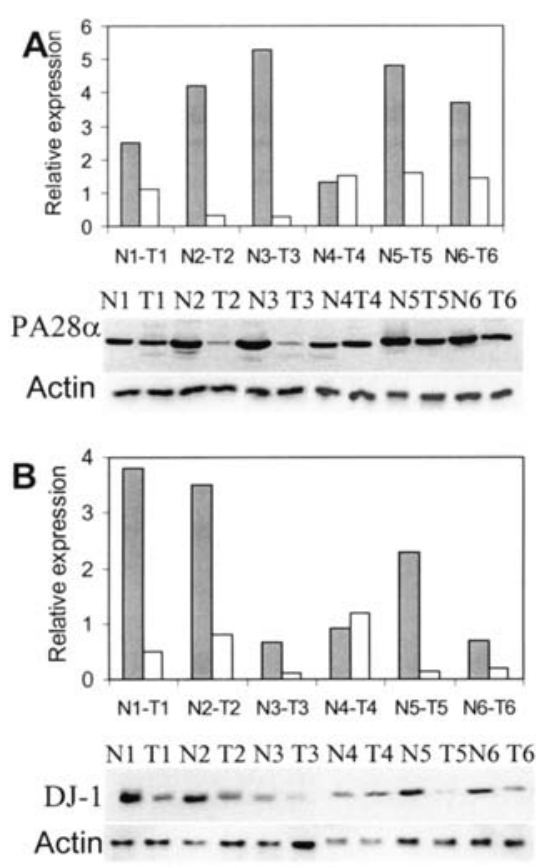

Figure 3. Western blotting of PA28 $\alpha$ and DJ-1 in 6 pairs of HBV-infected, well-differentiated HCC tumour and adjacent non-tumour tissues. Proteins from each of the six pairs of tissue samples were separated by SDS-PAGE $(12 \%)$ and transferred to PVDF membranes. Proteins were immunodetected using the respective primary antibodies and HRP-conjugated secondary antibody. Signals were captured with the Multi Genius bio imaging system and signal intensity was analyzed by the GeneTools software (Syngene). Average relative expression levels $( \pm \mathrm{SD})$ in triplicate experiments were normalized to the level of $\beta$-actin.

and PA28 $\alpha$ were reduced by $>3$-fold $(\mathrm{p}<0.01)$ in tumours, compared to the non-tumour tissues. 

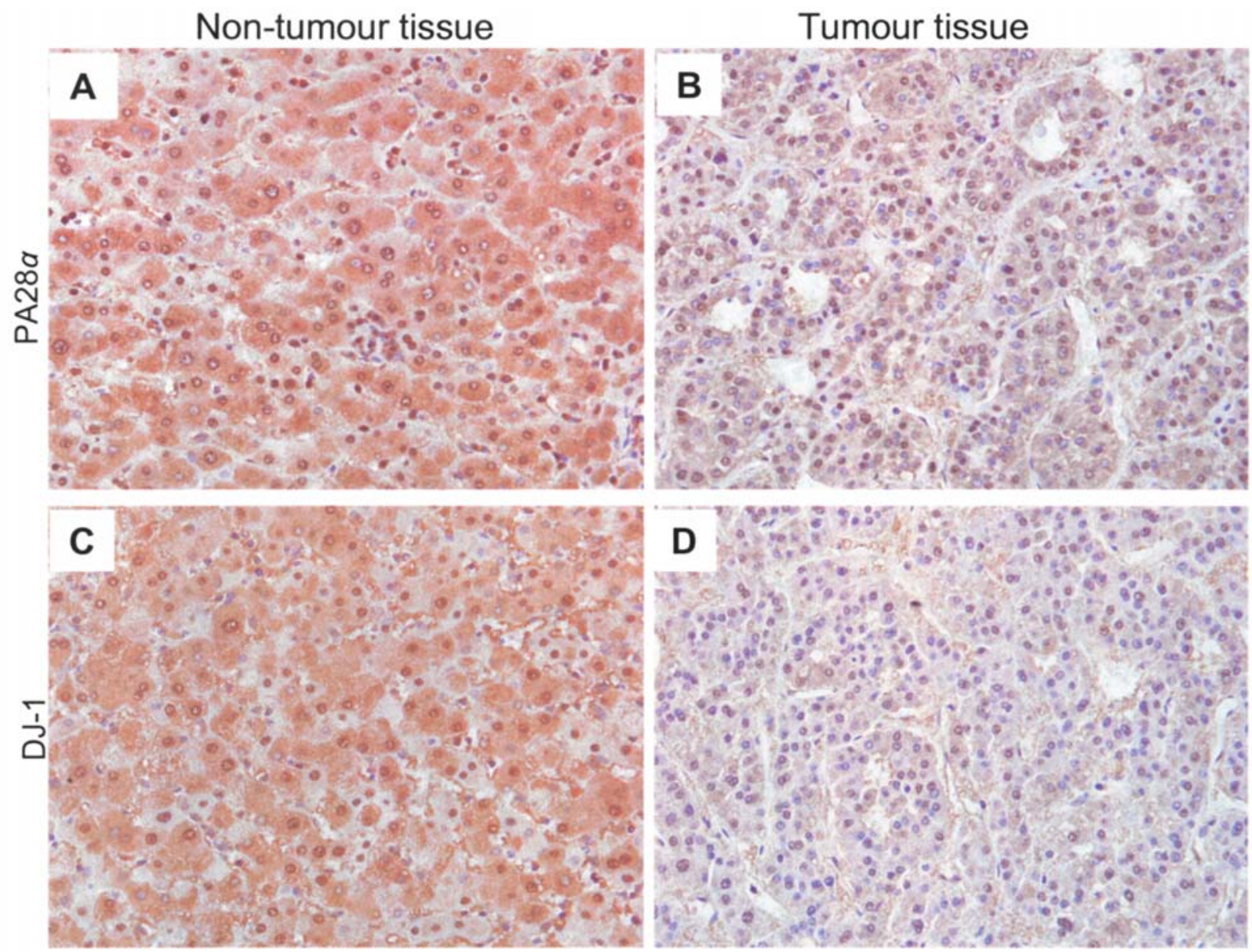

Figure 4. Representative immunostaining of PA28 $\alpha$ and DJ-1 in HBV-infected, well-differentiated HCC tumour and adjacent non-tumour tissue sections. Paraffin-embedded sections were deparaffinized in xylene and rehydrated in graded alcohols. After unmasking antigen and removing endogenous peroxidases, sections were incubated for $1 \mathrm{~h}$ with antibody against PA28 $\alpha$ and DJ-1 at room temperature, followed by detection with labelled dextran polymer conjugated with peroxidase and $\mathrm{DAB}^{+}$-substrate chromagen solution. Nuclei were lightly stained with Mayer's hematoxylin. The extent of staining was visually scored according to the staining intensity of cells. PA28 $\alpha$ and DJ-1 were strongly stained in the non-tumour tissue cells (A and C), and weakly stained in tumour cells (B and D). Original magnification x400.

Table III. Summary of immunohistochemical staining of DJ-1 and PA28 $\alpha$ in 15 pairs of paraffin-embedded, HBV-infected G1 hepatocellular carcinoma tissues.

Staining Non-tumor Tumor tissues Chi-square $\mathrm{p}$-value ${ }^{\mathrm{b}}$ intensity $^{\mathrm{a}}$ tissues $(\mathrm{n}=15) \quad(\mathrm{n}=15)$

\section{DJ-1}

\begin{tabular}{lccc} 
Negative & 0 & 8 & \\
Positive & 15 & 7 & 0.002 \\
PA28 $\alpha$ & & & \\
Negative & 0 & 6 & 0.017 \\
Positive & 15 & 9 & \\
\hline
\end{tabular}

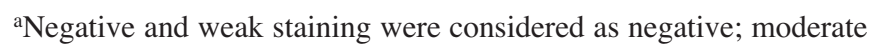
and strong staining were considered as positive. ${ }^{b}$ Fisher's exact test.

In situ expression of PA28 $\alpha$ and DJ-1 was also examined in 15 paraffin-embedded $\mathrm{HBV}$-infected $\mathrm{G} 1$ tumour and their adjacent non-tumour tissues. A representative immunostaining of DJ-1 and PA28 $\alpha$ in paired tissues is shown in Fig. 4. As shown in Table III, all the 15 matched non-tumour tissues showed moderate to strong cytoplasmic staining of both DJ-1 and PA28 $\alpha$. However, PA28 $\alpha$ was negatively stained in 6 of 15 tumour tissues $(\mathrm{p}=0.017)$, whereas $\mathrm{DJ}-1$ was negatively stained in 8 of 15 tumour tissues $(\mathrm{p}=0.002)$. Therefore, DJ-1 and PA28 $\alpha$ were down-regulated in most of the tested HBVinfected G1 tumours, compared to their adjacent non-tumour tissues.

\section{Discussion}

$\mathrm{HCC}$ is one of the most common cancers in the world and causes approximately one million deaths every year. Underlying the early molecular events of tumour onset may help to improve the diagnostic efficiency and develop more effective therapeutic strategies against HCC. With the aid of the proteomic approach integrated with a sophisticated bioinformatic tool and MALDI-TOF MS and MS/MS, we have identified 15 differentially expressed proteins from the HBVinfected G1 tumours. These proteins could be functionally interrelated and linked to the initiation of hepatocarcinogenesis. They were classified into several functional groups including 
metabolism, signal transduction, apoptosis and metastasis (Table II). Although the majority of proteins identified in our study have been reported $(4,8,9,21)$, several other proteins, e.g. DJ-1 and PA28 $\alpha$, have not previously been reported to be differentially expressed in HCC. In this study, we focused on these two proteins and their differential expression was further verified by immunoblotting and immunohistochemistry in a panel of HBV-infected G1 tumours. Although there is a need to further verify the reduced expression of PA28 $\alpha$ and DJ-1 in more HBV-infected G1 tumours, and to investigate whether it is the same case in HCV-infected HCC tumours, the abnormal expression of these molecules and the involved biochemical pathways may elucidate, at least in part, the early events causing tumorigenesis in HBV-related HCC.

PA28 $\alpha$ is an activator in the immunoproteasome system which has a pivotal role in the control of the immune response. PA28 $\alpha$ enhances the generation of class I binding peptides by altering the cleavage pattern of the proteasome and is required for efficient antigen processing. Previous studies showed that PA28 $\alpha$ responded to viral or bacterial infection and was upregulated in the livers of lymphocytic choriomeningitis virusinfected mice (22), and its expression induced the production of HLA-class I in IFN-treated colon cancer cell line DLD-1 (23). The impaired expression of PA28 $\alpha$ was also reported in cervical carcinoma lesions (24). However, HER-2/neu overexpression in fibroblasts reduced the level of MHC class I surface antigens by impairing the expression of PA28 $\alpha$ and PA28ß and other proteasome components, thereby resulting in an immune escape phenotype of oncogene-transformed cells (25). Thus, a reduced level of PA28 $\alpha$ may favour the tumour progression. In HCC, a lower level of PA28 $\alpha$, as demonstrated by immunoblotting and IHC (Figs. 2-4), in HBV-infected G1 may indicate an early decreased immune response to transformation and onset of tumour formation. Clinically, we proposed that the level of PA28 $\alpha$ could be used as an immunological indicator, together with histological analysis, to assess the hepatocarcinogenesis.

DJ-1 is an integral mitochondrial protein that may have important functions in regulating mitochondrial physiology. It interacts, as an oncoprotein, with Daxx and inhibits apoptosis signal-regulating kinase 1 activity and cell death (26). Several lines of evidence suggested that DJ-1 played a role in tumorigenesis in breast cancer, non-small cell lung carcinoma and prostate cancer (27-29). Moreover, DJ-1 positively regulates p53 through Topors-mediated sumoylation (30), and is recognized as a key negative regulator of PTEN that may be a useful prognostic marker for cancer (31). Sumoylation on a lysine residue at K130 and K130 mutation abrogated all of the functions of DJ-1, including ras-dependent transformation, cell growth promotion and anti-UV-induced apoptosis activities (32). Currently, Pardo et al found that DJ-1 was highly expressed and secreted in the malignant melanoma cells and considered as a potential serum biomarker in patients (33). In patients with breast cancer, DJ-1 was detected in the serum of $37 \%$ as a circulating tumour antigen (27). From our study, we observed the down-regulation of DJ-1 in the early stage of HBV-infected HCC (Figs. 2-4). The reduced level of DJ-1 may indicate the abnormal pathophysiological state of mitochondria and mitochondrial disease initiates the tumorigenesis in HBV-infected HCC. As it has been described as a circulating tumour antigen in serum from $37 \%$ of patients with breast cancer (27), we will study whether this is the case in $\mathrm{HBV}$-infected $\mathrm{HCC}$ to further assess its clinical value as an early diagnostic biomarker in patients.

In summary, we present here the comparative analysis of proteome profiles from $\mathrm{HBV}$-infected G1 tumour and adjacent non-tumour tissues, and identify the differentially expressed molecules which are potentially responsible for the early onset of tumorigenesis and are valuable markers for early diagnosis in patients with HCC. We show the involvement of molecules in a variety of pathways, including signal transduction, metabolism and anti-apoptosis in the early stage of HCC. Although we have only validated our results in a limited number of clinical specimens, the reduced expression of the molecules, PA28 $\alpha$ and DJ-1, could be a promising novel biomarker for malignancy detection and diagnosis in HBVinfected HCC.

\section{Acknowledgements}

This work was supported in part by the Academic Research Fund (R-179-000-032-112) from the National University of Singapore.

\section{References}

1. Chen CJ and Chen DS: Interaction of hepatitis B virus, chemical carcinogen, and genetic susceptibility: multistage hepatocarcinogenesis with multifactorial etiology. Hepatol 36: 1046-1049, 2002 .

2. Fujiyama S, Tanaka M, Maeda S, Ashihara H, Hirata R and Tomita K: Tumor markers in early diagnosis, follow-up and management of patients with hepatocellular carcinoma. Oncology 62: 57-63, 2002 .

3. Li C, Hong Y, Tan YX, et al: Accurate qualitative and quantitative proteomic analysis of clinical hepatocellular carcinoma using laser capture microdissection coupled with isotope-coded affinity tag and two-dimensional liquid chromatography mass spectrometry. Mol Cell Proteomics 3: 399-409, 2004.

4. Lee IN, Chen CH, Sheu JC, et al: Identification of human hepatocellular carcinoma-related biomarkers by two-dimensional difference gel electrophoresis and mass spectrometry. J Proteome Res 4: 2062-2069, 2005.

5. Luk JM, Lam CT, Siu AF, et al: Proteomic profiling of hepatocellular carcinoma in Chinese cohort reveals heat-shock proteins (Hsp27, Hsp70, GRP78) up-regulation and their associated prognostic values. Proteomics 6: 1049-1057, 2006.

6. Zeindl-Eberhart E, Haraida S, Liebmann S, et al: Detection and identification of tumor-associated protein variants in human hepatocellular carcinomas. Hepatol 39: 540-549, 2004.

7. Yokoyama Y, Kuramitsu Y, Takashima M, et al: Proteomic profiling of proteins decreased in hepatocellular carcinoma from patients infected with hepatitis C virus. Proteomics 4: 2111-2116, 2004.

8. Kim W, Lim S, Kim JS, et al: Comparison of proteome between hepatitis B virus- and hepatitis $\mathrm{C}$ virus-associated hepatocellular carcinoma. Clin Cancer Res 9: 5493-5500, 2003.

9. Liang CR, Leow CK, Neo JC, et al: Proteome analysis of human hepatocellular carcinoma tissues by two-dimensional difference gel electrophoresis and mass spectrometry. Proteomics 5: 2258-2271, 2005.

10. Park KS, Cho SY, Kim H and Paik YK: Proteomic alterations of the variants of human aldehyde dehydrogenase isozymes correlate with hepatocellular carcinoma. Int J Cancer 97: 261-265, 2002.

11. Feng JT, Liu YK, Song HY, et al: Heat-shock protein 27: a potential biomarker for hepatocellular carcinoma identified by serum proteome analysis. Proteomics 5: 4581-4588, 2005.

12. Takashima M, Kuramitsu Y, Yokoyama Y, et al: Overexpression of alpha enolase in hepatitis $\mathrm{C}$ virus-related hepatocellular carcinoma: association with tumor progression as determined by proteomic analysis. Proteomics 5: 1686-1692, 2005. 
13. Lee IN, Chen $\mathrm{CH}$, Sheu JC, et al: Identification of complement $\mathrm{C} 3 \mathrm{a}$ as a candidate biomarker in human chronic hepatitis $\mathrm{C}$ and $\mathrm{HCV}$-related hepatocellular carcinoma using a proteomics approach. Proteomics 6: 2865-2873, 2006.

14. Ding SJ, Li Y, Tan YX, et al: From proteomic analysis to clinical significance: overexpression of cytokeratin 19 correlates with hepatocellular carcinoma metastasis. Mol Cell Proteomics 3: 73-81, 2004.

15. Kuramitsu Y, Harada T, Takashima M, et al: Increased expression and phosphorylation of liver glutamine synthetase in welldifferentiated hepatocellular carcinoma tissues from patients infected with hepatitis C virus. Electrophoresis 27: 1651-1658, 2006.

16. Nissom PM, Lo SL, Lo JC, et al: Hcc-2, a novel mammalian ER thioredoxin that is differentially expressed in hepatocellular carcinoma. FEBS Lett 580: 2216-2226, 2006.

17. Iizuka N, Oka M, Yamada-Okabe H, et al: Comparison of gene expression profiles between hepatitis B virus- and hepatitis $\mathrm{C}$ virus-infected hepatocellular carcinoma by oligonucleotide microarray data on the basis of a supervised learning method. Cancer Res 62: 3939-3944, 2002.

18. Desmet VJ, Gerber M, Hoofnagle JH, Manns M and Scheuer PJ: Classification of chronic hepatitis: diagnosis, grading and staging. Hepatol 19: 1513-1520, 1994.

19. Zhang D, Tai LK, Wong LL, Sethi SK and Koay ES: Proteomics of breast cancer: enhanced expression of cytokeratin 19 in human epidermal growth factor receptor type 2 positive breast tumors. Proteomics 5: 1797-1805, 2005.

20. Zhang D, Tai LK, Wong LL, Chiu LL, Sethi SK and Koay ESC: Proteomic study reveals that proteins involved in metabolic and detoxification pathways are highly expressed in HER-2/neupositive breast cancer. Mol Cell Proteomics 4: 1686-1696, 2005.

21. Li C, Tan YX, Zhou H, et al: Proteomic analysis of hepatitis B virus-associated hepatocellular carcinoma: Identification of potential tumor markers. Proteomics 5: 1125-1139, 2005.

22. Khan S, van den Broek M, Schwarz K, de Giuli R, Diener PA and Groettrup M: Immunoproteasomes largely replace constitutive proteasomes during an antiviral and antibacterial immune response in the liver. J Immunol 167: 6859-6868, 2001.
23. Miyagi T, Tatsumi T, Takehara T, Kanto T, Kuzushita N, Sugimoto $\mathrm{Y}$, et al: Impaired expression of proteasome subunits and human leukocyte antigens class I in human colon cancer cells. J Gastroenterol Hepatol 18: 32-40, 2003.

24. Ritz U, Momburg F, Pilch H, Huber C, Maeurer MJ and Seliger B: Deficient expression of components of the MHC class I antigen processing machinery in human cervical carcinoma. Int J Oncol 19: 1211-1220, 2001 .

25. Herrmann F, Lehr HA, Drexler I, et al: HER-2/neu-mediated regulation of components of the MHC class I antigen-processing pathway. Cancer Res 64: 215-220, 2004.

26. Junn E, Taniguchi H, Jeong BS, Zhao X, Ichijo H and Mouradian MM: Interaction of DJ-1 with Daxx inhibits apoptosis signal-regulating kinase 1 activity and cell death. Proc Natl Acad Sci USA 102: 9691-9696, 2005.

27. Le Naour F, Misek DE, Krause MC, et al: Proteomics-based identification of RS/DJ-1 as a novel circulating tumor antigen in breast cancer. Clin Cancer Res 7: 3328-3335, 2001.

28. MacKeigan JP, Clements CM, Lich JD, Pope RM, Hod Y and Ting JP: Proteomic profiling drug-induced apoptosis in non-small cell lung carcinoma: identification of RS/DJ-1 and RhoGDIalpha. Cancer Res 63: 6928-6934, 2003.

29. Hod Y: Differential control of apoptosis by DJ-1 in prostate benign and cancer cells. J Cell Biochem 92: 1221-1233, 2004.

30. Shinbo Y, Taira T, Niki T, Iguchi-Ariga SM and Ariga H: DJ-1 restores p53 transcription activity inhibited by Topors/p53BP3. Int J Oncol 26: 641-648, 2005.

31. Kim RH, Peters M, Jang Y, et al: DJ-1, a novel regulator of the tumor suppressor PTEN. Cancer Cell 7: 263-273, 2005.

32. Shinbo Y, Niki T, Taira T, et al: Proper SUMO-1 conjugation is essential to DJ-1 to exert its full activities. Cell Death Differ 13: 96-108, 2006.

33. Pardo M, Garcia A, Thomas B, et al: The characterization of the invasion phenotype of uveal melanoma tumour cells shows the presence of MUC18 and HMG-1 metastasis markers and leads to the identification of DJ-1 as a potential serum biomarker. Int J Cancer 119: 1014-1022, 2006. 